Case Report

\title{
Autologous Dermis Graft Implantation: A Novel Approach to Reinforcement in Giant Hiatal Hernias
}

\author{
Balázs Kovács $\mathbb{D}^{1},{ }^{1}$ Mikolt Orosz, ${ }^{1}$ Máté Csucska, ${ }^{1}$ Saurabh Singhal $\mathbb{D},{ }^{2}$ Árpád Juhász, \\ and Zoltán Lóderer ${ }^{1}$ \\ ${ }^{1}$ Department of Vascular, Plastic and General Surgery, Markusovszky University Teaching Hospital, Szombathely, Hungary \\ ${ }^{2}$ Norton Thoracic Institute, St. Joseph's Hospital and Medical Center, Phoenix, AZ, USA \\ Correspondence should be addressed to Balázs Kovács; email@kovacsbalazs.com
}

Received 15 January 2018; Accepted 24 April 2018; Published 8 May 2018

Academic Editor: Gabriel Sandblom

Copyright (C) 2018 Balázs Kovács et al. This is an open access article distributed under the Creative Commons Attribution License, which permits unrestricted use, distribution, and reproduction in any medium, provided the original work is properly cited.

\begin{abstract}
Objectives. Nonreinforced tensile repair of giant hiatal hernias is susceptible to recurrence, and the role of mesh graft implantation remains controversial. Creating a new and viable choice without the use of high-cost biological allografts is desirable. This study presents the application of dermis graft reinforcement, a cost-efficient, easily adaptable alternative, in graft reinforcement of giant hiatal hernia repairs. Methods. A 62-year-old female patient with recurrent giant hiatal hernia $(9 \times 11 \mathrm{~cm})$ and upside down stomach, immediately following the Belsey repair done in another department, was selected for the pilot procedure. The standard three-stitch nonabsorbable reconstruction of diaphragmatic crura was undertaken via laparoscopic approach. A $12 \times 6 \mathrm{~cm}$ dermis autograft was harvested from the loose abdominal skin. "U" figure onlay reinforcement of diaphragm closure was secured with titanium staples. The procedure was completed with a standard Dor fundoplication. One- and seven-month follow-ups were conducted. Results. No short-term postoperative complications were observed. One-month follow-up showed normal anatomical location of abdominal viscera on computed tomography imaging. High-resolution manometry showed normal lower esophageal sphincter pressure. Preoperative abdominal complaints were resolved. Procedural costs were lower than the average cost following mesh graft reinforcement. Conclusion. Dermis graft reinforcement is a cheap, easily adaptable procedure in the repair of giant hiatal hernias, even in the setting of laparoscopic reoperative procedure.
\end{abstract}

\section{Introduction}

As the number of surgical interventions required for gastroesophageal reflux disease (GERD) and/or hiatal hernias is increasing worldwide, surgeons encounter increasingly more complicated and inveterate cases with large hiatal defects. The number of publications per year related to hiatal repairs has almost doubled in the last decade (Figure 1).

Pearson et al., in their classification of hiatal hernias in 1983, defined giant hiatal defect as the herniation of $30 \%$ or more of the stomach into the thoracic cavity in the posterior mediastinum [1]. Koch et al. later defined large hernias as the ones with the surface area of hiatal defect larger than $5 \mathrm{~cm}^{2}$ [2]. Laparoscopic repair of giant hiatal hernias presents a new challenge in the operative treatment. These defects are highly susceptible to recurrence, if repaired in a nonreinforced tensile manner [3]. The aim of this study was to find a feasible and safe technique to reinforce this hiatal gap.

\section{Methods}

A 62-year-old female patient, with a history of complication during the reversal of anesthesia following varicectomy procedure, underwent repeated evaluations for complaints of dyspnoea on exertion and epigastric discomfort while seated. The symptoms aggravated upon bending forward. The patient had a BMI of $28 \mathrm{~kg} / \mathrm{m}^{2}$. After dubious chest X-ray findings, computed tomography (CAT) evaluation showed an "upside-down stomach" situated in the thorax with an organoaxial rotation, along with a $9 \times 11 \mathrm{~cm}$ hiatal defect. Endoscopy showed LA-B erosive esophagitis and confirmed the nonanatomical location of the gastroesophageal junction. The patient underwent a Belsey Mark IV antirefluxplasty 


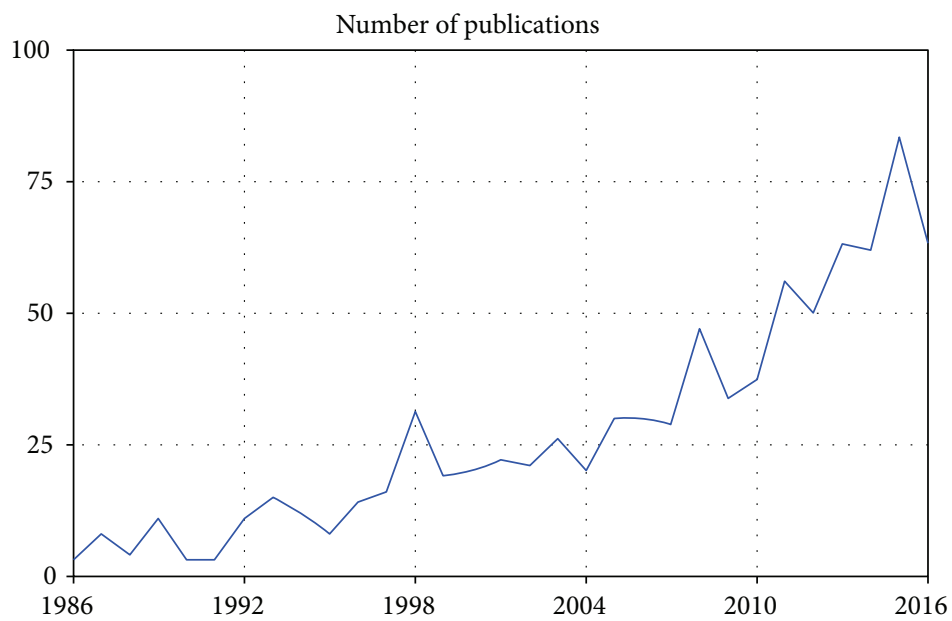

Figure 1: Number of publications per year on PubMed search for keywords "hiatal hernia repair."

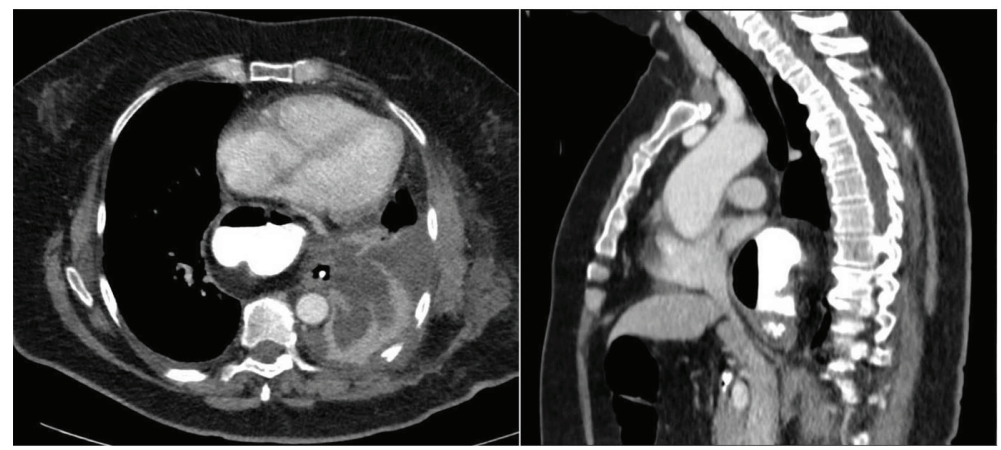

FIgURE 2: Preoperative CAT images show paraoesophageal herniation of the antrum into the thoracic cavity.

with hiatal reconstruction through left posterolateral thoracotomy at a thoracic surgery institute. On the 6th postoperative day, the patient developed severe dysphagia. Repeat CAT imaging confirmed paraesophageal reherniation of the antrum (Figure 2), and the patient was referred to our institute for laparoscopic reoperative intervention.

Based on CAT scan findings, there was a high suspicion that the standard redo fundoplication with primary hiatal closure will be insufficient, and reinforcement of the crural closure will be necessary. In view of the debatable role of synthetic mesh reinforcement, our department evaluated the possibility of autologous dermis graft implementation, as we have utilized this graft in parastomal hernias. There is reemerging evidence in Hungarian as well as international literature for the clinical use in complicated incisional hernias [4-6], this experience has supported the effectivity of autologous dermis graft as reinforcement. After board review, the procedure protocol was approved by the Regional Research Ethics Committee. Following discussion with the patient and thorough explanation of the medical procedure, informed consent was obtained for dermis graft harvesting and implantation.

The laparoscopic exploration was done on the 12th postoperative day. There was failure of the diaphragmatic crural stitches along with herniation of the antrum, pylorus, and greater omentum into the thorax (Figure 3(a)). We removed the second line of Belsey reconstruction that fixed the fundoplication to the left diaphragmatic crus. The lower esophageal sphincter (LES) was located in an intra-abdominal position, and the esophagus required no more mediastinal mobilization. After mobilization of the greater curvature, the hiatal defect was closed using 3 stitches of 0 braided nonabsorbable suture. Suture line seemed to be under tension, requiring reinforcement. Loose skin of the left lower abdomen was excised, thoroughly deepithelized, and cleaned from fatty tissue. The graft was rolled up and introduced into the abdomen through a $10 \mathrm{~mm}$ trocar. The graft was placed covering the suture line, also surrounding the esophagus in a Ushaped form, and was fixed to the diaphragm using titanium staples (Figures $3(\mathrm{l})$ and $3(\mathrm{~m})$ ), essentially resulting in a tensile repair with an onlay allograft reinforcement. The antireflux operation was completed creating an anterior (Dor) fundoplication around the distal esophagus. After desufflation, the abdominal skin defect was primarily closed with a running suture line. The total operative time was 195 minutes, including the preparation of the dermis graft which took 27 minutes.

\section{Results}

After an uneventful postoperative period, the patient was discharged on a pasty diet on the 3rd postoperative day. 


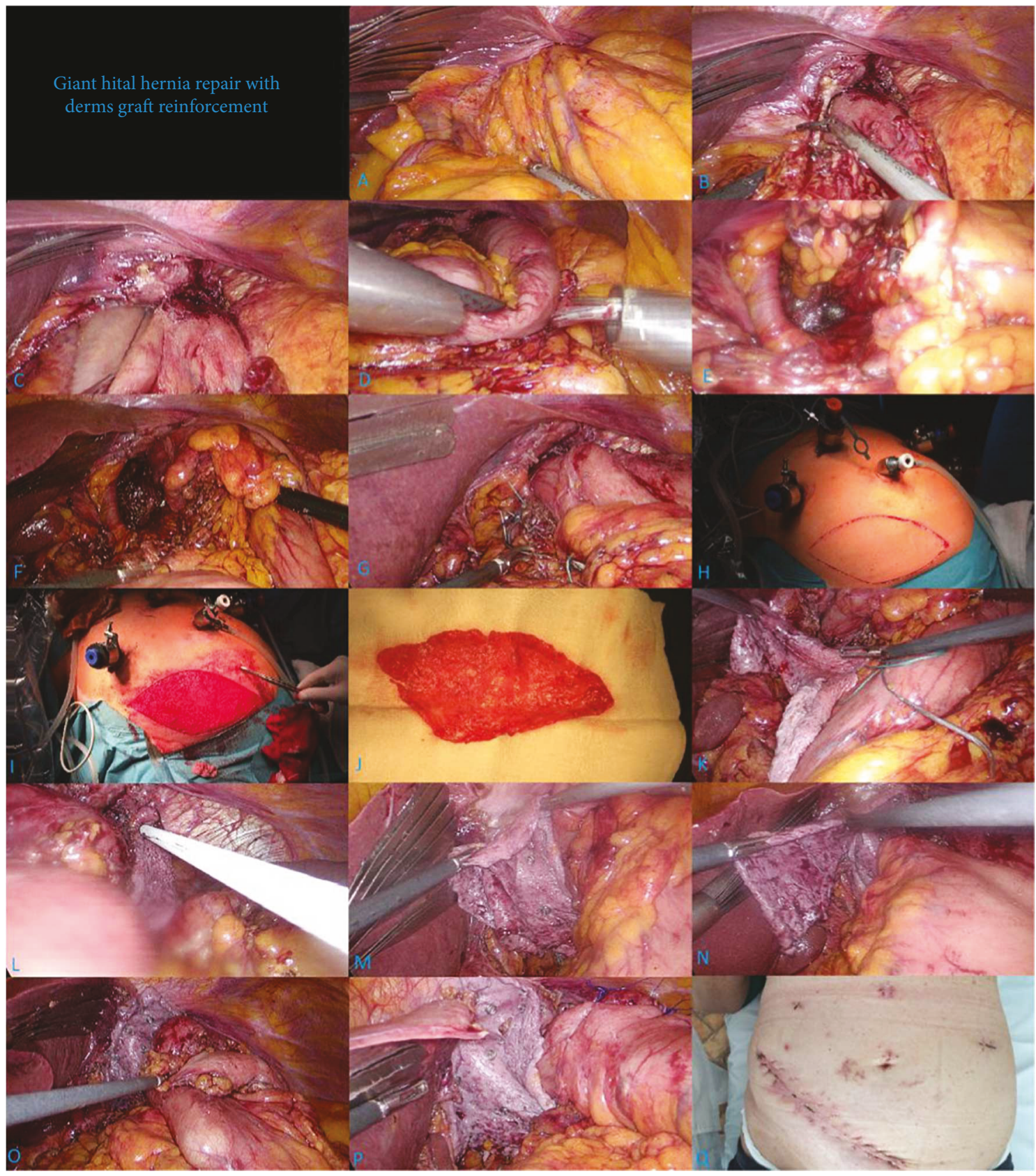

FIGURE 3: Surgical steps for giant hiatal hernia repair with dermis graft reinforcement: (a) initial intraoperative finding during redo surgery, $60 \%$ of the stomach is in the thoracic cavity; (b) adhesiolysis; (c) retraction of the mobile lesser curvature into the abdominal cavity; (d) stomach in the intra-abdominal position; (e) visualization of the crural stitch failure; (f) Belsey rear suture line left in place, fundoplication adequately mobilized; (g) suture repair of the defect; (h) harvesting site for dermis graft; (i) deepithelized skin flap; (j) dermis graft free of adipose tissue; (k) graft introduced into the abdomen; (l) graft fixation to the left diaphragmatic crura; (m) graft fixation to the right diaphragmatic crura; (n) excess graft removed; (o) anterior Dor's fundoplication; (p) final position of fundoplication wrap and the reinforced hiatal repair; (q) scar at 1-month follow-up.

The patient was asymptomatic and doing well at the 14day and 1-month follow-ups. The thoracoabdominal CAT scan at one-month follow-up showed normal anatomical position of the abdominal viscera (Figure 4(a)). Esophageal high-resolution manometry (HRM) revealed satisfactory LES function with adequate esophageal motility. The patient did not report any epigastric discomfort, and the self-perceived physical fitness was improved.
Further follow-up was conducted 7 months postoperatively. Gastroduodenoscopy showed no signs of reflux, reherniation, or failure of fundoplication. HRM validated the continuous low-pressure zone at LES position. Repeat CAT scan at 7 months reassured normal anatomical position of viscera (Figure 4(b)). The patient was free of any abdominal or chest complaints. At 12-month telephone follow-up, the patient had no GI complaints and no 


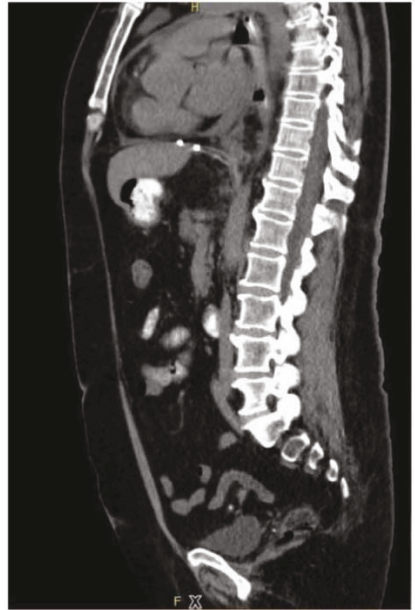

(a)
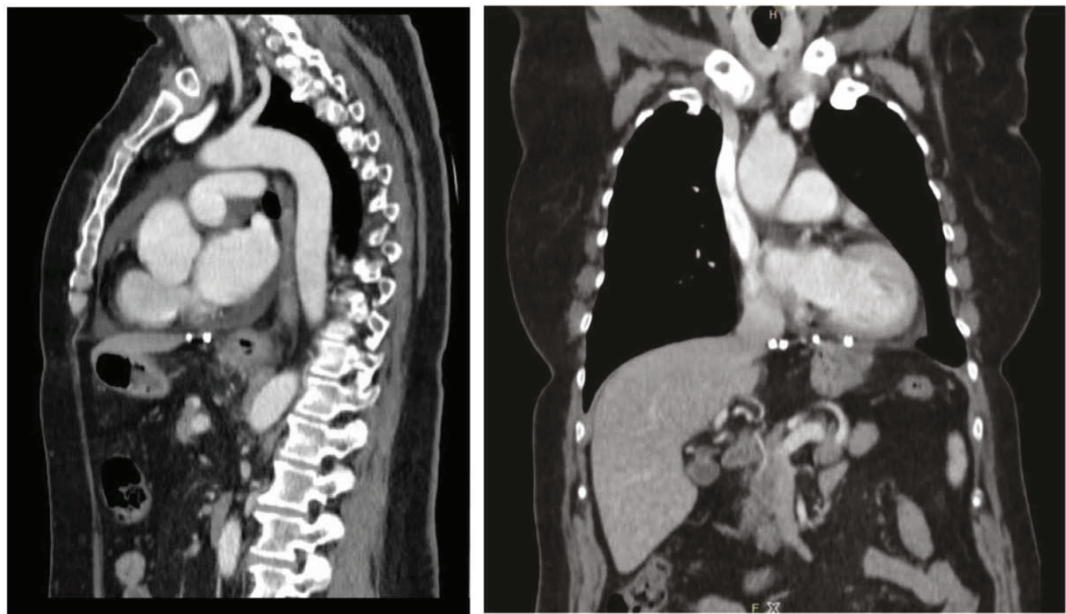

(b)

FIgURE 4: (a) CAT scan at 1-month follow-up shows anatomical position of abdominal viscera. (b) CAT scan at 7-month follow-up: sagittal and coronal reconstruction.

respiratory complaints on exertion. There were minimal additional costs only due to the relatively longer operative time. Additional costs were significantly lower than the cost of available biological matrices.

\section{Discussion}

There is a building evidence favoring the need for reinforcement in the repair of giant hiatal hernias. The failure rate following the repair of large hiatal hernias without further reinforcement of the reconstructed diaphragm is unacceptably high [7]. Besides consistent esophageal and ventricular mobilization, a number of special interventions have been readily published to reduce recurrence rate. If the short esophagus is encountered intraoperatively, creating a Collis gastroplasty is justified. This has been reported to reduce the rate of recurrence [8]. As in the inguinal hernia repair, the mesh reinforcement was one of the earliest techniques to improve outcomes following a hiatal hernia repair. However, it carries a small but potentially fatal risk of complications in the long run. Mesh migration is one of these recognized complications and can further lead to stricture, perforation, or fistula formation of the esophagus or the wrap [9]. While the use of synthetic mesh prosthesis could lower the recurrence rate to an acceptable 12\% [9], potentially severe complications cast a shadow on the generalized use of synthetic prosthesis, as life-threatening complications are far worse tolerated in the attendance of a benign disease [10-17]. After publication of these complications associated with the synthetic mesh graft, a notable number of novel approaches have emerged using various biological implants for reinforcement [18].

Bell et al. proposed an acellular dermal matrix allograft (with human dermal collagen derivative) reinforcement of the sutured hiatus using suture fixation, with results that encouraged further research [19]. The newer biological prostheses, however, further augment the cost of the hiatal repair [20]. Sasse et al. reduced the cost of reinforced hiatal hernia repair by using urinary bladder matrix for reinforcement, but the additional graft cost was still over $1000 \mathrm{USD} /$ case [21]. Bjelovic et al. utilized autologous fascia lata graft for the reinforcement of the sutured diaphragmatic crura. They reported no recurrence in their 10 case series [22].

Autologous dermal grafts were one of the earliest biological matrices used in hernia repair in the early 20th century [23]. While there were numerous attempts of whole skin utilization as well [24], it was clarified in animal research that the complete and thorough deepithelization of the implanted graft not only facilitates incorporation but also mitigates infectious complications [25]. Shaffer presented a series of 27 massive inguinal and ventral hernia cases with dermal graft augmentation in 1956, where he also gave anecdotal mention of dermal graft use in a paraesophageal hernia case [26]. With the advancement in implantable synthetic material technology, the use of dermal grafts disappeared from international literature for the latter half of the 20th century. The use of autologous dermal graft in ventral and incisional hernias has reemerged in the last ten years, as the rates of recurrence and morbidity in complicated hernias remain high despite the use of synthetic meshes, and the use of biological matrices heavily augments procedural costs. Deepithelized dermal autograft has been used with promising results in these intricate cases [4-6]. As aforementioned literature presents, the implantation of dermal grafts into the structures of the abdominal wall can be considered safe.

Despite rapidly growing literature, there is still no gold standard for the type of graft to be used for reinforcement, in order to achieve the lowest rates of recurrence, complications, and procedural costs in giant hiatal hernias [10]. We present a novel approach to the reinforcement of hiatal hernia repair using a biological graft implant, with the benefits of allograft utilization. The increase in overall costs with our approach is only due to the increased operative time and consequent anesthetic costs. The method 
seems to have only advantages: graft is autologous with no risk of rejection; dermis is soft and pliable without risk of usuration or fistula formation of the esophageal wall; and there is no risk of severe visceral adhesions. The operative procedure is easily applicable and reproducible and requires routine laparoscopic instruments only. Numerous methods of deepithelization are utilized in regular plastic surgical work; one example is part of Schwarzmann breast reduction; the method applied should not affect the procedure as long as results is an even surfaced, completely epithelium, and fatty tissue-free graft.

The only mentionable disadvantage is the scar formation at the harvesting site. This can be minimized with a proper suturing and wound care. If patient composition permits, the optimal harvesting site is the loose skin of the lower abdomen. However, some patients with giant hiatal hernia often experience difficulty of oral intake resulting in a decreased $\mathrm{BMI}$; in these cases, consultation with a plastic surgeon is recommended.

While comparing the additional burden of an abdominal skin scar to high-cost alternative grafts might be troublesome, the biological graft alternatives [20,21] are not covered by the Hungarian State Insurance and therefore could be utilized only if purchased by the patient, and that is their major limiting factor in a number of socialized medical systems. The dermis graft thus has the advantage of being a viable, low-cost, and acceptable alternative to these patients. There is an ethical dilemma that lies within comparing the possible severe esophageal complications of nonbiological grafts, the high cost of biological implantables, and the scar formation of dermis graft harvesting. As all these three present in very different modalities, it should be entrusted to the thoroughly informed and enlightened patient to choose from the options available.

Our department has utilized the autologous dermis graft in primal reconstruction of giant hiatal hernias in three more cases which reconfirm the excellent short-term results; data collection for prospective case series is in progress.

\section{Conclusions}

We suggest onlay dermis graft reinforcement of the sutured diaphragmatic repair as a viable alternative in giant hiatal hernia repair. The method is eligible for further clinical evaluation. Further patient recruitment and long-term follow-up would be beneficial to determine the place of dermis graft reinforcement among the use of other biological matrices.
Abbreviations
CAT: Computed tomography
GERD: Gastroesophageal reflux disease
HRM: High-resolution manometry
LES: $\quad$ Lower esophageal sphincter.

\section{Data Availability}

Data are available through the corresponding author.

\section{Conflicts of Interest}

Drs. Balázs Kovács, Mikolt Orosz, Máté Csucska, Saurabh Singhal, Árpád Juhász, and Zoltán Lóderer have no conflicts of interest or financial ties to disclose.

\section{Authors' Contributions}

Balázs Kovács acquired, analyzed, and interpreted the data and drafted the manuscript. Mikolt Orosz and Máté Csucska were the assistant surgeons in the pilot procedure. Saurabh Singhal revised the manuscript. Árpád Juhász was the operating surgeon in the pilot procedure and the study supervisor, interpreted the data, and drafted and revised the manuscript. Zoltán Lóderer provided the study idea and did critical revision of the manuscript for important intellectual content.

\section{References}

[1] F. G. Pearson, J. D. Cooper, R. Ilves, T. R. J. Todd, and W. R. E. Jamieson, "Massive hiatal hernia with incarceration: a report of 53 cases," The Annals of Thoracic Surgery, vol. 35, no. 1, pp. 45-51, 1983.

[2] O. O. Koch, M. Schurich, S. A. Antoniou et al., "Predictability of hiatal hernia/defect size: is there a correlation between preand intraoperative findings?," Hernia, vol. 18, no. 6, pp. 883888, 2014.

[3] S. A. Antoniou, G. A. Antoniou, O. O. Koch, R. Pointner, and F. A. Granderath, "Lower recurrence rates after meshreinforced versus simple hiatal hernia repair: a meta-analysis of randomized trials," Surgical Laparoscopy, Endoscopy \& Percutaneous Techniques, vol. 22, no. 6, pp. 498-502, 2012.

[4] G. Martis and L. Damjanovich, "Use of double-layer autologous dermal flap in the treatment of recurrent and/or infected incisional hernias: presentation of the surgical technique and the results of 1-year follow-up-a prospective, consecutive cohort study," Hernia, vol. 20, no. 3, pp. 461-470, 2016.

[5] T. D. Samson, E. W. Buchel, and P. B. Garvey, "Repair of infected abdominal wall hernias in obese patients using autologous dermal grafts for reinforcement," Plastic and Reconstructive Surgery, vol. 116, no. 2, pp. 523-527, 2005.

[6] G. Martis and L. Damjanovich, "The role of autografts in the treatment of complicated incisional hernias," Magyar Sebészet, vol. 69, no. 2, pp. 45-53, 2016.

[7] M. Hashemi, J. H. Peters, T. R. DeMeester et al., "Laparoscopic repair of large type III hiatal hernia: objective followup reveals high recurrence rate," Journal of the American College of Surgeons, vol. 190, no. 5, pp. 553-560, 2000.

[8] J. D. Luketich, K. S. Nason, N. A. Christie et al., "Outcomes after a decade of laparoscopic giant paraesophageal hernia repair," The Journal of Thoracic and Cardiovascular Surgery, vol. 139, no. 2, pp. 395-404.e1, 2010.

[9] B. P. Müller-Stich, H. G. Kenngott, M. Gondan et al., "Use of mesh in laparoscopic Paraesophageal hernia repair: a metaanalysis and risk-benefit analysis," PLoS One, vol. 10, no. 10, article e0139547, 2015.

[10] J. R. Huddy, S. R. Markar, M. Z. Ni et al., "Laparoscopic repair of hiatus hernia: does mesh type influence outcome? A metaanalysis and European survey study," Surgical Endoscopy, vol. 30, no. 12, pp. 5209-5221, 2016. 
[11] E. Virgilio, P. Mercantini, and M. Cavallini, "Partial transmural gastroesophageal migration of polypropylene mesh after surgery for a recurrent hiatal hernia," European Review for Medical and Pharmacological Sciences, vol. 20, no. 17, pp. 3515-3516, 2016.

[12] L. Bognár, Ö. P. Horváth, J. Solt, G. Jancsó, and A. Vereczkei, "Intraesophageal mesh migration following laparoscopic hiatal hernia repair," Magyar Sebészet, vol. 68, no. 4, pp. 176-180, 2015.

[13] V. Porziella, A. Cesario, F. Lococo et al., "Complete transmural gastric migration of PTFE mesh after surgery for a recurrent hiatal hernia," European Review for Medical and Pharmacological Sciences, vol. 16, Supplement 4, pp. 42-43, 2012.

[14] M. Carpelan-Holmström, O. Kruuna, J. Salo, L. Kylänpää, and T. Scheinin, "Late mesh migration through the stomach wall after laparoscopic refundoplication using a dual-sided PTFE/ePTFE mesh," Hernia, vol. 15, no. 2, pp. 217-220, 2011.

[15] R. P. Tatum, S. Shalhub, B. K. Oelschlager, and C. A. Pellegrini, "Complications of PTFE mesh at the diaphragmatic hiatus," Journal of Gastrointestinal Surgery, vol. 12, no. 5, pp. 953957, 2008.

[16] A. Juhasz, A. Sundaram, M. Hoshino, T. H. Lee, and S. K. Mittal, "Outcomes of surgical management of symptomatic large recurrent hiatus hernia," Surgical Endoscopy, vol. 26, no. 6, pp. 1501-1508, 2012.

[17] Ö. P. Horváth, G. Varga, Z. Biró, A. Papp, L. Bognár, and A. Vereczkei, "Complications and reoperations following laparoscopic antireflux surgery," Magyar Sebészet, vol. 69, no. 3, pp. 91-99, 2016.

[18] S. A. Antoniou, R. Pointner, F.-A. Granderath, and F. Köckerling, "The use of biological meshes in diaphragmatic defects - an evidence-based review of the literature," Frontiers in Surgery, vol. 2, p. 56, 2015.

[19] R. C. W. Bell, J. Fearon, and K. D. Freeman, “Allograft dermal matrix hiatoplasty during laparoscopic primary fundoplication, paraesophageal hernia repair, and reoperation for failed hiatal hernia repair," Surgical Endoscopy, vol. 27, no. 6, pp. 1997-2004, 2013.

[20] M. Hiles, R. D. Record Ritchie, and A. M. Altizer, "Are biologic grafts effective for hernia repair?: A systematic review of the literature," Surgical Innovation, vol. 16, no. 1, pp. 26-37, 2008.

[21] K. C. Sasse, D. L. Warner, E. Ackerman, and J. Brandt, "Hiatal hernia repair with novel biological graft reinforcement," JSLS : Journal of the Society of Laparoendoscopic Surgeons, vol. 20, no. 2, article e2016.00016, 2016.

[22] M. Bjelovic, T. Babic, B. Spica, D. Gunjic, M. Veselinovic, and V. Bascarevic, "The use of autologous fascia lata graft in the laparoscopic reinforcement of large hiatal defect: initial observations of the surgical technique," BMC Surgery, vol. 15, no. 1, p. 22, 2015.

[23] O. Loewe, "Uber Hautimplantationen an Stelle der Freien Faszienplastick," Munchener Medizininische Wochenschrift, vol. 24, article 13201, 1913.

[24] G. B. Mair, "Preliminary report on the use of whole skingrafts as a substitute for fascial sutures in the treatment of herniæ," British Journal of Surgery, vol. 32, no. 127, pp. 381-385, 1945.
[25] E. Rehn, "Das Kutane and Sunkutane Bindegewebe als Plstisches Material," Munchener Medizininische Wochenschrift, vol. 61, p. 118, 1914.

[26] J. Shaffer, "Massive and recurrent hernias; use of dermal grafts in carrying out repair," California Medicine, vol. 85, no. 1, pp. $10-14,1956$. 


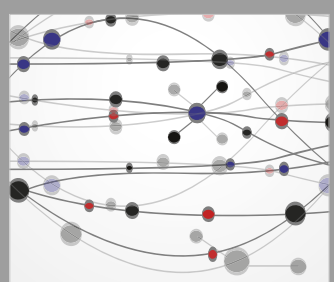

The Scientific World Journal
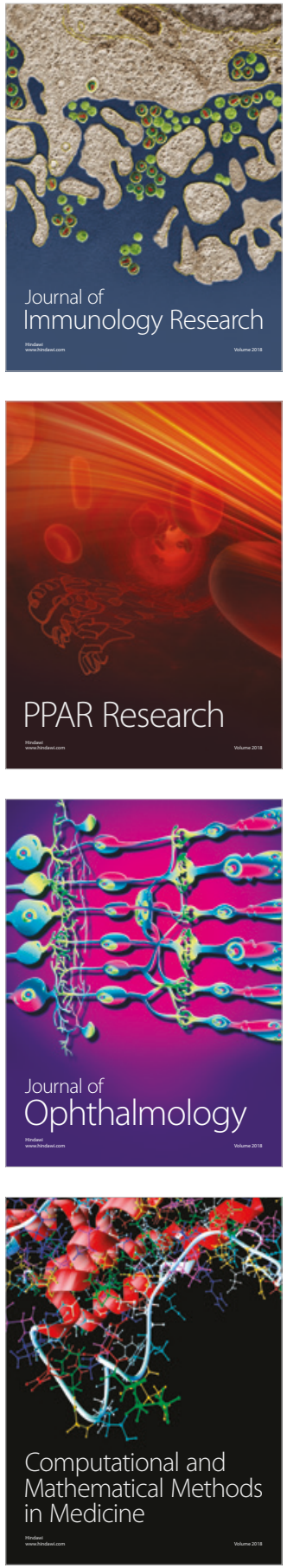

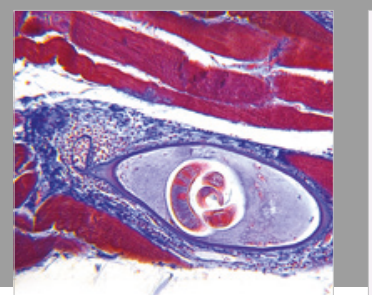

Gastroenterology Research and Practice

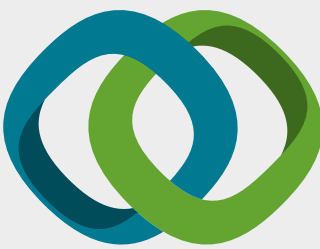

\section{Hindawi}

Submit your manuscripts at

www.hindawi.com
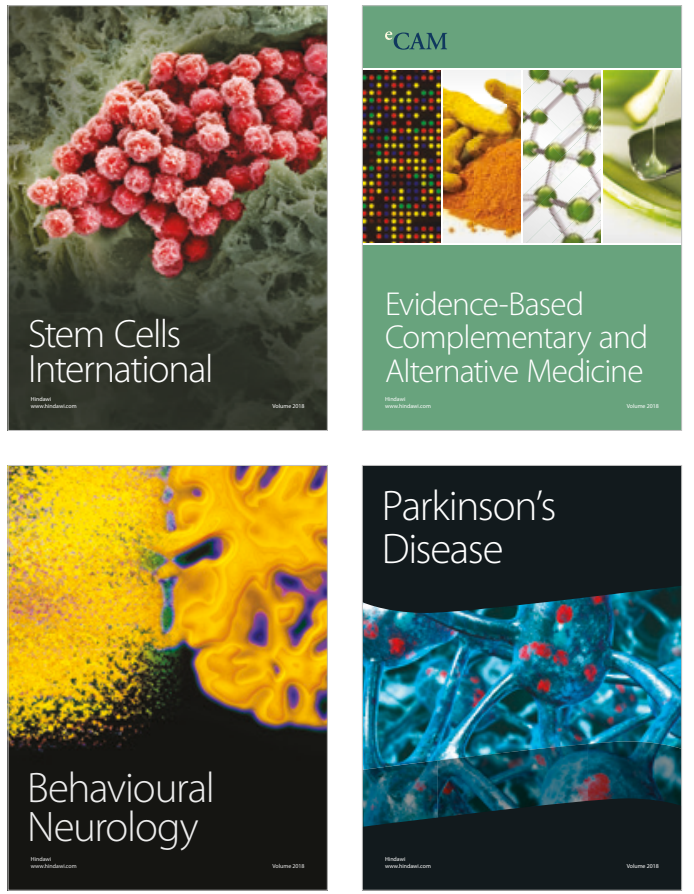

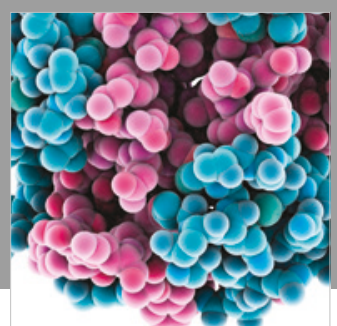

ournal of

Diabetes Research

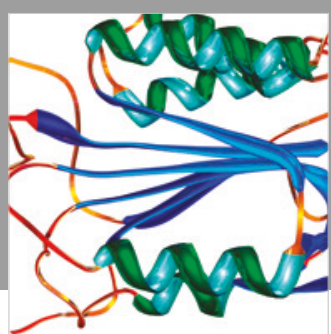

Disease Markers
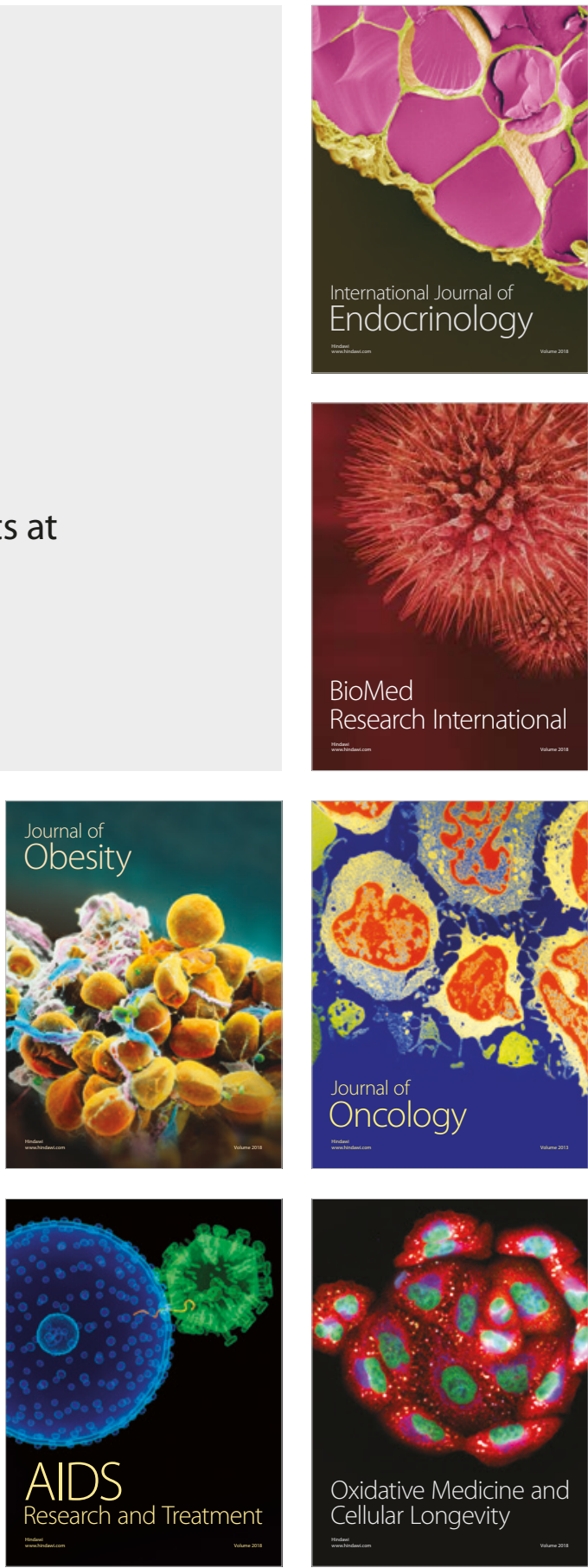